Produto \& Produção, vol. 11, n. 2, p. 67 - 84, jun. 2010

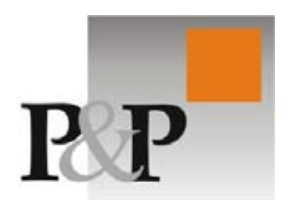

\title{
Desenvolvendo Indicadores de Produtividade e Qualidade em Hospitais: Uma Proposta de Método
}

\author{
Alberto W. Ramos, Dr. \\ Departamento de Engenharia de Produção da Escola Politécnica da USP \\ awramos@usp.br \\ Dario Ikuo Miyake, Ph. D. \\ Departamento de Engenharia de Produção da Escola Politécnica da USP \\ dariomiy@usp.br
}

\begin{abstract}
RESUMO
Este artigo contempla a necessidade de tornar o processo de gestão mais integrado e efetivo na busca de melhores resultados em organizações da área da saúde, nas quais a prática da avaliação de desempenho ainda não está tão amplamente disseminada. Para isso, propõe um método para estabelecer indicadores de produtividade e qualidade em hospitais, com base em conceitos da gestão por processos e abordagem sistêmica. O método proposto foi aplicado em uma pesquisa-ação realizada no contexto de um grande hospital que vem empreendendo esforços para se firmar como um centro de referência promovendo a melhoria de seus padrões de atendimento. O trabalho explicita como um sistema de indicadores de desempenho deve ser criado a partir da identificação dos processos-chave da organização. Ao final, fornece um exemplo de aplicação mostrando como o método proposto estrutura a sequência dos passos requeridos para a definição, seleção e medição destes indicadores.
\end{abstract}

Palavras-chave: indicadores, gestão por processos, hospital.

\section{INTRODUÇÃO}

Hospitais, como qualquer outra empresa, buscam criar indicadores para melhor gerenciar seu negócio. Embora haja alguns indicadores comumente empregados para se fazer comparações ou benchmarking entre estas instituições, muitas vezes eles não são criados ou selecionados a partir de um método estruturado que permita identificar quais são os aspectos críticos a serem monitorados e, eventualmente, aprimorados nos processos de prestação de serviço que oferecem. 
Este artigo tem por objetivo propor um método de simples aplicação que permita identificar, com base nos conceitos de gestão por processo, quais são os indicadores necessários para gestores de hospitais. Ele fornece um conjunto de passos que abrange desde a identificação da missão de cada processo, até a definição dos indicadores-chave. Ao final do artigo é apresentado um exemplo de sua aplicação.

\section{O HOSPITAL VISTO COMO UM CONJUNTO DE PROCESSOS}

À semelhança do que ocorre em outros setores da economia e da sociedade, no setor da saúde, de uma maneira geral, os hospitais têm adotado o modelo tradicional de organização por função, ou seja, dividindo atividades e recursos por áreas ou unidades de negócio voltadas a determinadas funções. Esta visão fragmentada traz vários problemas, tais como: dificuldade de integração entre as unidades; surgimento de problemas de coordenação que, quando envolvem mais de uma área, tornam-se complexos; existência de lacunas ou sobreposições nas atividades executadas; problemas de comunicação; busca de objetivos dissociados que podem não contribuir para o alcance de bons resultados nos processos de atendimento.

Harrigton et al. (1997) e Tenner e DeToro (1997) evidenciam ser esta forma de organização inadequada e ultrapassada, e propõem que se estabeleça a gestão por processos como um modo de superar estes problemas. A base desta colocação é a visão do negócio como um sistema, ou seja, um todo composto de partes (ou os processos) que se inter-relacionam, de forma a fazer com que os objetivos propostos sejam atingidos da melhor forma possível, evitando sub-otimizações ou desperdícios de recursos.

Já na década de 1980, autores consagrados da área de gestão e qualidade como Deming (1982) e Juran (1988) ressaltavam a necessidade desta visão sistêmica para a melhoria da qualidade dos produtos de uma organização, sejam eles bens ou serviços. Posteriormente, Senge (1994) retomou a importância da abordagem sistêmica para a promoção da aprendizagem organizacional. Para Gadd e Oakland (1996), a promoção da visão sistêmica das organizações também fundamenta outros conceitos, como cadeias de clientes e de fornecedores, e abordagens gerenciais, como trabalho em equipe (teamwork) e reengenharia de processos (business process reengineering), que ganharam evidência na década de 1990. Num trabalho mais recente, Hellsten e Klefsjö (2000) salientaram a importância de considerar de forma sistêmica não apenas a organização que for o objeto de análise, mas também o próprio modo de implementação das intervenções que se revelarem necessárias para assegurar a efetividade das mesmas.

Entendendo-se processo como um conjunto de atividades executadas com um propósito determinado, todo trabalho executado numa empresa como um hospital pode ser enxergado como sendo um processo ou parte de um processo maior. Assim sendo, na análise e no gerenciamento de processos hospitalares, é imprescindível adotar-se a abordagem sistêmica, o que implica em procurar conhecer as inter-relações entre os componentes existentes no sistema e as pessoas que nele trabalham (Churchman, 1972).

Um processo é constituído de atividades que, a partir de insumos obtidos de certos fornecedores, criam saídas que têm valor para os clientes. A Figura 1 mostra 
que uma empresa pode ser enxergada como um conjunto de processos e que cada processo, em geral, atravessa diferentes departamentos para cumprir a função esperada pelos clientes. Assim, enquanto as áreas funcionais estruturam organizacionalmente a cadeia de comando, os processos cruzam estas diferentes áreas gerando fluxos transversais de materiais, pessoas, serviços ou informações.

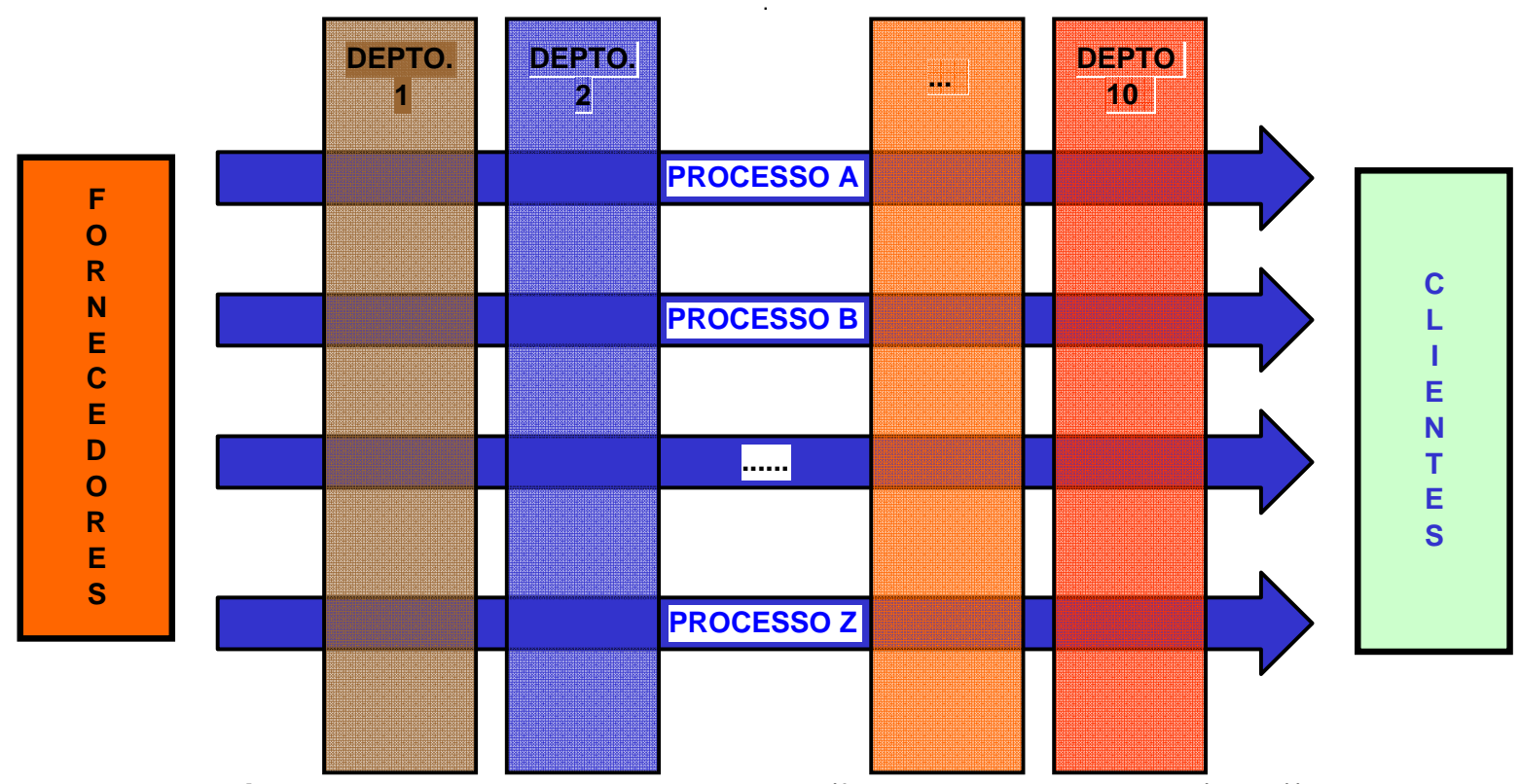

Figura 1 - Processos x Departamentos (fonte: Tenner e DeToro (1997))

A Figura 2 apresenta, genericamente, a hierarquia de processos existentes numa organização. Ela sugere que um processo pode ser entendido como uma combinação de sub-processos, que cada sub-processo pode ser dividido em atividades menores e que estas podem ser desagregadas em tarefas mais específicas. Isso faz com que o resultado final de um processo dependa do modo como suas partes se inter-relacionam e são gerenciadas.

As Figuras 1 e 2 evidenciam a importância de os agentes envolvidos nas partes que constituem os processos desempenharem de forma efetiva as tarefas, atividades ou sub-processos para alcançar-se os resultados esperados, independentemente de pertencerem a um mesmo departamento ou não.

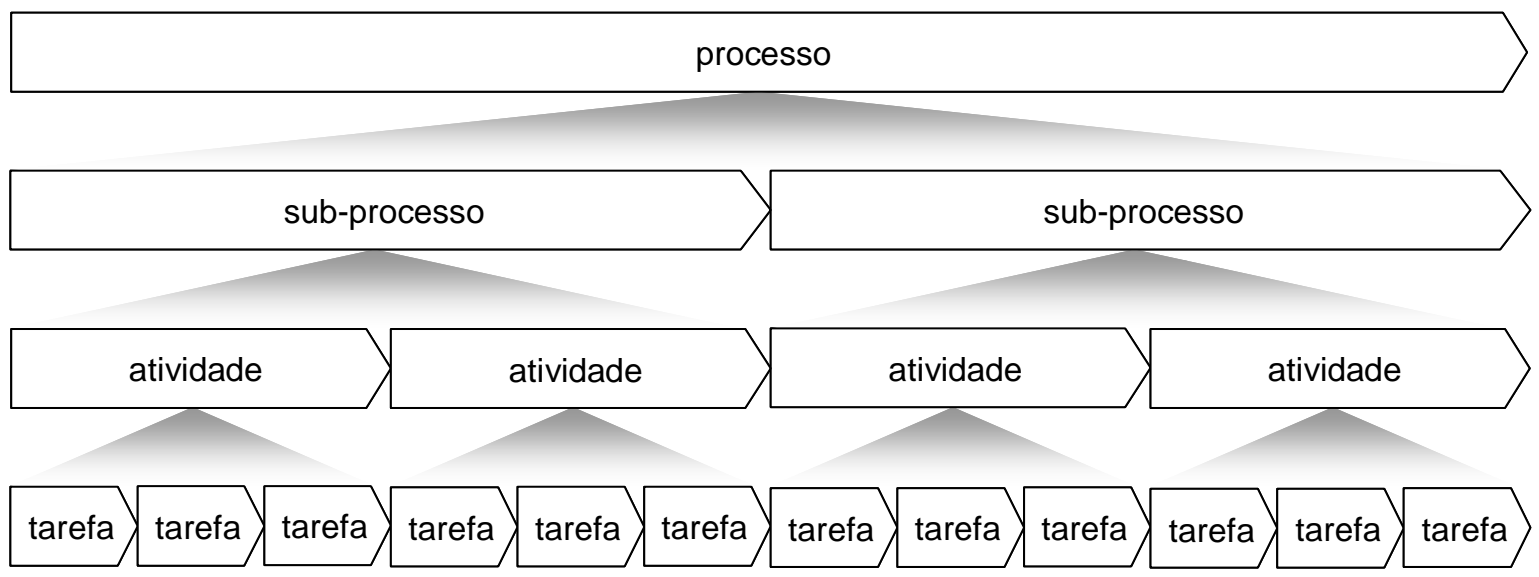

Figura 2 - Hierarquia de Processos numa Organização (elaborada pelos autores) 


\section{PRODUTIVIDADE E QUALIDADE EM PROCESSOS HOSPITALARES}

Em um estudo sobre a relação entre gestão de processos e qualidade em saúde, Bittar (2000) observa que os processos hospitalares necessitam ser avaliados e controlados quanto a variáveis como eficácia, eficiência, produção, produtividade e qualidade, quanto à prevenção e redução da morbimortalidade e quanto à sua imagem perante usuários ou clientes.

A despeito da relevância desta questão, mesmo em países como os Estados Unidos, existem estudos que admitem que a prática de medição e monitoramento de processos na área da saúde ainda é pouco desenvolvida, assim como a cultura de promover a melhoria de processos com base em sua avaliação sistemática (Loeb, 2004; Williams et al., 2005). Os autores destes estudos argumentam que, como a falta de métodos de medição baseados em evidências limita o potencial de melhoria em hospitais, algumas iniciativas no sentido de desenvolver e de disseminar um sistema de avaliação com indicadores padronizados passaram a ser implementadas nos Estados Unidos envolvendo a participação de diversas associações, instituições e órgãos públicos da área de saúde. Vale destacar um destes programas que foi conduzido com foco na avaliação da qualidade de determinados tipos de tratamento de saúde sob os auspícios da Joint Commission on Accreditation of Healthcare Organizations (JCAHO). Mais de 3000 hospitais participaram do projeto piloto deste programa recebendo feedback sobre seu desempenho ao longo de dois anos e demonstraram que, em geral, conseguiram atingir melhorias significativas nos tratamentos considerados.

No Brasil, alguns estudos sugerem que há uma conscientização crescente sobre a importância do uso de indicadores de produtividade (BITTAR, 1996) e qualidade (KOTAKA et al., 1997) visando a melhoria do desempenho de processos hospitalares. A Portaria n. ${ }^{0}$ 1101/GM, do Ministério da Saúde de 12 de junho de 2002, a qual estabelece os parâmetros assistenciais do Sistema Único de Saúde (SUS), define "parâmetros de produtividade" como aqueles destinados a estimar a capacidade de produção dos recursos, equipamentos e serviços de assistência à saúde, sejam eles humanos, materiais ou físicos, salientando sua importância como instrumentos de planejamento, controle, regulação e avaliação do SUS. Em relação a recursos humanos, esta portaria preconiza, por exemplo, como capacidade de produção para o enfermeiro 03/consultas/hora e, para o médico, 04/consultas/hora, não fazendo distinção entre consulta nova e consulta de seguimento, mas ressaltando que esses dados podem variar de acordo com convenções sindicais, dissídios coletivos das categorias profissionais ou adoção, pelo gestor, de políticas específicas. Visando a promoção da avaliação de serviços de saúde, a Agência Nacional de Vigilância Sanitária (ANVISA) tem preconizado a adoção de indicadores padronizados, o que facilitaria comparações de dados e informações dentro dos hospitais e entre hospitais. Na Tabela 1 são apresentados alguns dos indicadores preconizados que têm como foco a produtividade. 
Tabela 1. Indicadores para avaliação hospitalar (elaborada pelos autores).

\begin{tabular}{lcr}
\hline \multicolumn{1}{c}{ indicador } & acrônimo & definição \\
\hline $\begin{array}{l}\text { Taxa de produtividade } \\
\text { hospitalar }\end{array}$ & TxPH & $\begin{array}{r}\text { [(Número de internações/ano } \times \text { Média de permanência } \times \\
\text { Número de leitos existentes/ano) / 365 dias) } \times 100\end{array}$ \\
$\begin{array}{l}\text { Taxa de ocupação } \\
\text { Taxa média de } \\
\text { permanência }\end{array}$ & TxOH & $\begin{array}{r}\text { (Número de pacientes-dia em um período / Número de } \\
\text { leitos-dia em um mesmo período) } \times 100\end{array}$ \\
\hline
\end{tabular}

Segundo Schiesari e Kisil (2003), a preocupação em avaliar instituições hospitalares no Brasil data da década de 1940, mas somente na década de 1990, com o advento do programa de organização da acreditação no Brasil, é que ações mais efetivas surgiram nesta direção. Isso contribuiu para que diversas associações e órgãos da área de saúde convergissem para a idéia de se adotar um manual único a ser seguido no país. A elaboração da versão inicial do Manual Brasileiro de Acreditação em 1998 foi fruto deste esforço que culminou na formação da Organização Nacional de Acreditação (ONA). Em sua versão de 2006, este manual preconiza, como um requisito fundamental do padrão de gestão das principais áreas de serviços hospitalares, a adoção efetiva de uma sistemática de medição e avaliação de processos (ANVISA-ONA, 2006).

Apesar deste processo de amadurecimento, pesquisas acadêmicas revelam que poucos serviços de saúde dispõem de indicadores para gestão hospitalar e menos ainda os utilizam (MALIK e TELES, 2001). Urge, portanto, desenvolver propostas que subsidiem o planejamento e a implementação de uma sistemática permanente de avaliação e melhoria tanto dos serviços de assistência à saúde como dos processos de administração nos hospitais, estimulando-os a atingirem padrões mais elevados de desempenho.

Embora diversas variáveis possam ser avaliadas no gerenciamento de processos, as iniciativas discutidas acima revelam que, assim como pode ser constatado em outros setores industriais, o binômio produtividade e qualidade também têm merecido destacada atenção no âmbito da gestão de processos hospitalares (NEPOTE, 2003; FELDMAN et al., 2005). Para a sua avaliação, vale salientar que, em qualquer processo, é fundamental contemplar os seguintes três pontos chave:

a) A eficiência: indica como o processo utiliza ou converte seus recursos (ou entradas) para a obtenção das saídas;

b) A eficácia: indica como o processo atinge os seus objetivos ou sua missão, que é a razão de sua existência;

c) Os resultados: indicam como o processo atende aos seus clientes, ou seja, as etapas posteriores ou outros processos (IBRAHIM, 2001).

Assim sendo, na criação de indicadores para o gerenciamento de um processo é importante abarcar todos estes pontos pela complementaridade que existe entre os mesmos e, assim, buscar uma visão mais sistêmica do desempenho do processo.

Segundo Sumanth (1984), a avaliação da produtividade no contexto de uma empresa pode ser tratada de um modo abrangente considerando sua produtividade total ou, de um modo parcial, a produtividade de somente determinado(s) tipo(s) de recurso. Em ambos os casos, a produtividade é fundamentalmente definida como a 
razão entre as saídas e entradas do sistema ou processo. Basicamente, a diferença entre a consideração da produtividade em um nível mais macro ou micro consiste no grau de agregação com que os valores de saídas e entradas são computados. Procurando relacionar a eficiência e a eficácia com a produtividade, Sumanth (1984) sugere que a primeira exerce grande influência sobre as entradas enquanto que as saídas são, fundamentalmente, influenciadas pela segunda. Assim, a produtividade seria determinada em função tanto da eficiência como da eficácia com que opera o sistema ou processo considerado.

Quando se fala de qualidade em um processo, esta tem basicamente duas dimensões: ausência de erros ou deficiências (JURAN, 1997) e cumprimento da missão do processo. Assim como a produtividade, a qualidade também é afetada pela eficiência e eficácia. A existência de erros compromete a eficiência do processo, uma vez que haverá retrabalho (ou reprocesso) das atividades executadas incorretamente, fazendo com que sejam gastos mais recursos do que se a atividade fosse, simplesmente, executada corretamente na primeira vez. Assim, a ocorrência de erros causa também um impacto negativo na produtividade.

Por outro lado, sob a perspectiva da eficácia do processo, considerar a qualidade somente como uma questão relacionada a erros é uma visão por demais simplista. Afinal, "nada errado" não significa necessariamente "tudo certo", ou seja, que a missão do processo tenha sido eficazmente alcançada.

Conseqüentemente, pode-se ter um processo que seja extremamente eficiente, mas que não seja eficaz. Isto é o que Crosby (1986) definia como "fazer certo as coisas erradas". Na Figura 3, são apresentados os componentes fundamentais da produtividade e da qualidade de um processo, bem como as influências exercidas pela eficiência e pela eficácia com que o mesmo opera. As relações representadas são gerais podendo ser consideradas tanto no contexto de processos de produção de bens como de serviços.

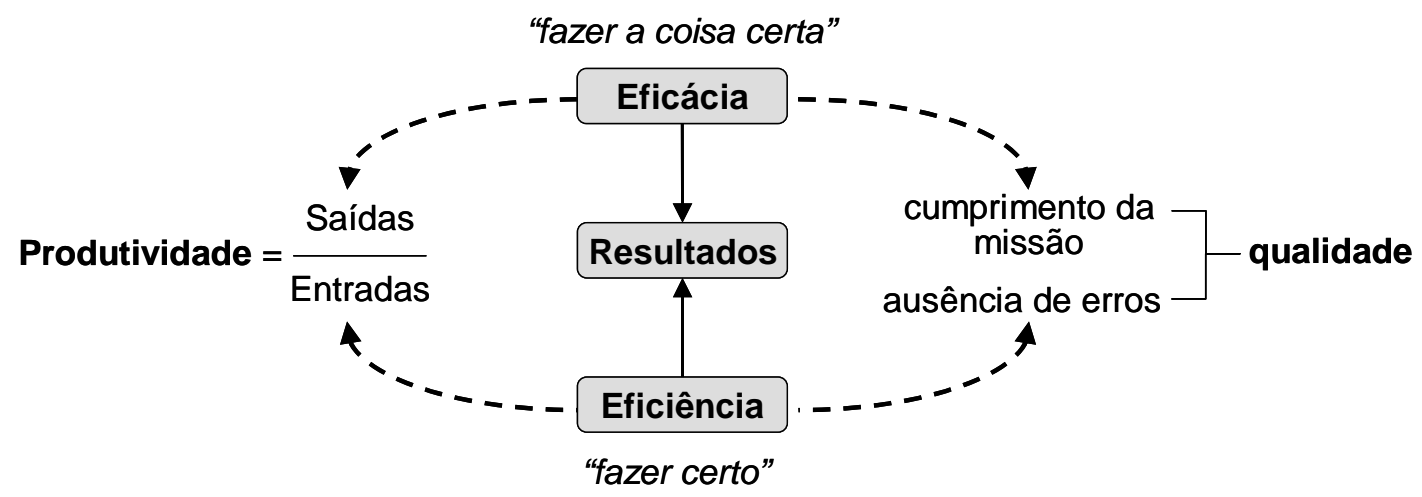

Figura 3 - Produtividade e qualidade de um processo (elaborada pelos autores)

Levando-se ainda em consideração o terceiro ponto-chave de um processo, o resultado, na Figura 3, (ele) é colocado como um efeito central que depende da eficiência e eficácia do processo relacionado. Assim, o resultado que está diretamente atrelado à satisfação dos clientes não pode ser plenamente alcançado se tanto a eficiência como a eficácia não forem satisfatórias. O impacto sobre o cliente é evidente: aumento de custos (e preços), processos não cumpridos nos prazos e não entrega do prometido. 
O presente trabalho foi desenvolvido no contexto de um grande hospital localizado na cidade de São Paulo, cuja direção vem empreendendo grandes esforços para se firmar como um centro de referência na área de oncologia, promovendo projetos de melhoria de seus padrões de atendimento, e que entendeu que o alcance deste objetivo precisa ser necessariamente sustentado por uma forma de gestão mais efetiva. Para isso, a direção do hospital criou um projeto com o propósito de construir um sistema de indicadores que pudesse sinalizar as necessidades de melhoria de processos de uma forma consoante com esta estratégia. Para o desenvolvimento deste projeto foi montada uma equipe com gestores internos e pesquisadores externos, incluindo os autores deste trabalho, e o seu desenvolvimento foi conduzido em quatro fases como seguem:

Fase 1: Definição do escopo do projeto de implantação de um sistema de gestão com base em indicadores

Fase 2: $\quad$ Concepção de um método para a construção de um sistema de indicadores em hospitais

Fase 3: $\quad$ Construção do sistema de indicadores aplicando-se o método proposto

Fase 4: Implantação do sistema de indicadores no processo de gestão do hospital.

As Fases 1 e 4 envolveram intrinsecamente decisões e ações sob a responsabilidade do próprio hospital. Já o planejamento e condução das Fases 2 e 3 ficaram sob a responsabilidade dos autores deste trabalho. A realização deste trabalho teve, portanto, como motivação, justamente a oportunidade de desenvolver e aplicar empiricamente um método para implantação da gestão por processos em organizações hospitalares. Assim, o método apresentado neste artigo resultou de uma pesquisa de natureza qualitativa realizada ao longo destas duas fases durante o primeiro semestre de 2008.

Para a condução da Fase 2, os pesquisadores, autores deste trabalho, inicialmente apoiaram-se em observações diretas e entrevistas para desenvolver um amplo entendimento da situação-problema (necessidade de implantação do gerenciamento por processos balizado num sistema de indicadores) no hospital. Esta investigação revelou que o sistema de informação do hospital já permitia que a direção obtivesse e monitorasse indicadores macro como os da Tabela 1 e que os gestores responsáveis pelas principais unidades da organização tinham estabelecido sistemas próprios de supervisão apoiados em relatórios que já incluiam diversos indicadores. Estes, na grande maioria dos casos, consistiam essencialmente de indicadores de apontamento de produção, utilização de recursos, fluxo de clientes e relacionamento com os mesmos evidenciando grande preocupação com o modo como os processos vêm desempenhando do ponto de vista da eficiência e produtividade. Constatou-se, portanto, que havia uma falta de indicadores que possibilitassem avaliar também a eficácia e a qualidade dos processos, sugerindo que os apontamentos e relatórios existentes eram relevantes para a administração do hospital, mas que permitiam apenas uma visão parcial do desempenho face ao quadro mais geral apresentado na Figura 3. 
Além disso, os diferentes relatórios de indicadores identificados mostraram que se tratava de ferramentas dispersas e sem clara vinculação com os resultados finais dos processos considerados, fornecendo uma visão da eficiência e da produtividade com que cada unidade operava, mas não possibilitando à direção estabelecer ações e prioridades para, efetivamente, desenvolver competências do hospital de natureza mais clínica e técnica.

Isso veio a corroborar a necessidade de implantação de um sistema de indicadores que fosse realmente aplicável à gestão por processos tendo como propósito final a elevação dos padrões de atendimento. A partir deste entendimento, os pesquisadores elaboraram uma proposta de método para a construção de um sistema de indicadores fundamentado em instrumentos já consagrados de planejamento e gestão de organizações que facilitasse a sua implantação e aplicação.

O método proposto tem, como passo inicial, a identificação do que é importante para o hospital e a definição de métricas-chave para medição do desempenho em relação ao mesmo. Este passo é direcionado à identificação dos chamados Fatores Críticos de Sucesso (ROCKART, 1979) a partir dos quais devem ser desdobrados os Indicadores Chave de Desempenho (EPSTEIN e MANZONI, 1998). Quanto à forma de uso dos indicadores selecionados, adotou-se, em linhas gerais, a abordagem de modelos de gerenciamento como a da Administração por Objetivos (DRUCKER, 1987) e do Gerenciamento pelas Diretrizes (AKAO, 1991). Estes instrumentos promovem a idéia da administração sistêmica para a busca de resultados pelo estabelecimento de compromissos negociados e acordados entre 0 nível local - das partes que constituem uma organização - e central; sendo que o primeiro focaliza as metas a serem atingidas enquanto o segundo contempla o "como" atingi-las.

$\mathrm{Na}$ Fase 3, o método proposto foi aplicado na construção do sistema de indicadores do hospital objeto de estudo, envolvendo a participação de seus gestores e corpo clínico, por meio da estratégia de pesquisa ação. A pesquisa ação é um método de pesquisa de base empírica, concebido e realizado em estreita associação com uma ação e/ou solução de um problema no qual o pesquisador teve um envolvimento direto e ativo (BERTO e NAKANO, 1998). Para Coughlan e Coghlan (2002), quando aplicada na área de gestão de operações, a pesquisa-ação proporciona um meio de colaboração entre gestores e pesquisadores na consideração de realidades que são tanto relevantes do ponto de vista gerencial como intelectualmente interessantes e, assim, possibilita um aprofundamento de conhecimentos que conduz à melhoria de práticas e à extensão de teorias.

Para a condução da Fase 3 , dada a necessidade dos pesquisadores interagirem com médicos, físicos, enfermeiros e administradores das diversas áreas que constituem o hospital, a diretoria designou um recurso interno para coordenar o agendamento de entrevistas e reuniões visando a coleta de dados para o desenvolvimento do sistema de indicadores proposto. No decorrer de um período de três meses, os pesquisadores realizaram uma seqüência de 10 visitas de cerca de 3 horas cada às diversas áreas do hospital. Nestas visitas, coube aos pesquisadores 0 papel de definir a agenda e conduzir os participantes à geração de resultados ou decisões requeridos para o avanço do projeto. A participação dos pesquisadores estendeu-se até a consolidação, validação e entrega do sistema de indicadores para o conjunto de macro-processos contemplado(s). 


\section{A PROPOSTA DE MÉTODO PARA CONSTRUÇÃO DE UM SISTEMA DE INDICADORES EM HOSPITAIS}

Nesta seção, é apresentada a proposta de método que resultou da Fase 2 de concepção. Para a construção e implantação da gestão por processos numa organização complexa e de grande porte em que uma miríade de atividades é realizada, é fundamental que, inicialmente, sejam identificados os macro-processos, ou seja, os poucos processos vitais responsáveis pelo cumprimento da missão da organização/empresa. Não basta somente identificá-los, é também fundamental entender como contribuem para a missão da organização como um todo. No âmbito de um hospital, a missão pode ser formulada tanto para a organização como um todo, como para cada departamento ou serviço. Em qualquer nível, o enunciado da missão deve explicitar, sem ambigüidade, quatro conceitos chave: "esta organização (ou parte dela) existe para oferecer tais produtos, com tais características, para tais clientes visando alcançar tais resultados". Vale salientar que as missões das "partes" devem manter uma estrita relação de coerência e complementaridade com a missão do "todo" (CECílIO, 2000).

Uma vez definido o conjunto de macro-processos a ser focado e sua missão, deve-se prosseguir à busca de bons indicadores de eficiência e eficácia. Para balizar esta busca, foi proposto um procedimento para obter-se dos gestores de cada processo as definições ou posições necessárias. O procedimento proposto consiste de seis passos os quais são descritos nas seguintes seções.

\subsection{Identificação das principais atividades desempenhadas pela área}

Normalmente, as pessoas envolvidas têm muita dificuldade em enxergar a organização como um conjunto de processos interligados, pois naturalmente, em sua rotina de trabalho, tendem a lidar, sobretudo, com atividades e responsabilidades circunscritas ao seu raio de ação. Para facilitar este levantamento, pode-se perguntar a cada gestor quais são as principais atividades desempenhadas em sua área. Invariavelmente, esta pergunta conduz aos principais processos envolvidos, muito embora seja necessário, posteriormente, confrontá-los com os das respostas de outros gestores, pois há o risco de um mesmo processo receber diferentes nomes. Além disso, é preferível que os sub-processos identificados separadamente por diferentes gestores, mas que estejam encadeados, sejam vistos como partes de um mesmo processo maior.

\subsection{Definição da importância das atividades desempenhadas}

O esclarecimento deste ponto tem como propósito identificar a "missão" do "processo" ao qual estão relacionadas, porém, sem (se) utilizar estas palavras, visto que podem causar diferentes interpretações por diferentes pessoas. Além disso, este questionamento também visa fazer com que o gestor defenda a importância relativa do processo em questão frente à de outros desempenhados pela sua área. 


\subsection{Definição das etapas básicas de cada uma destas atividades}

A idéia aqui é que cada gestor descreva, num nível um pouco maior de detalhe, cada um de seus principais processos. Foi sugerido que um máximo de cinco etapas fossem descritas - no nível de atividade ou tarefa conforme a Figura 2 - para evitar um grau de complexidade desnecessário nesta fase.

\subsection{Identificação dos tipos de erro que podem ocorrem em cada etapa}

Com base na idéia da ferramenta chamada de Análise do Modo e Efeito da Falha (FMEA), procurou-se relacionar, a cada etapa do processo, os tipos de erros que poderiam ocorrer. Isso é relativamente simples quando as pessoas envolvidas são exatamente aquelas que executam a etapa de processo considerada, pois sabem muito bem relacionar os problemas existentes. Como regra geral, também, não há muita dificuldade em associar-se cada possível erro à sua consequência.

\subsection{Priorização das ocorrências}

Mesmo pedindo para cada gestor listar apenas algumas poucas etapas fundamentais para cada um de seus processos (atividades), é comum que a quantidade dos possíveis erros enumerados seja muito grande podendo ser inviável controlar efetivamente todos. Isso torna necessário considerar uma forma adequada de priorizar aqueles que têm maior importância e motivou a aplicação da técnica GUTF por meio da qual as dimensões Gravidade (prejuízos causados), Urgência (necessidade de tomada de ação de correção), Tendência (evolução no tempo) e Frequência (incidência do problema) de cada tipo de erro devem ser avaliadas.

Para a execução deste passo, empregaram-se os critérios apresentados na Tabela 2 para avaliação de cada tipo de erro quanto às quatro dimensões consideradas com base numa escala de cinco pontos. A técnica GUTF permite priorizar os tipos de erros avaliados por uma nota calculada pelo produto dos pontos atribuídos a estas quatro dimensões. Quanto maior a nota do tipo de erro considerado, maior é a prioridade de controlá-lo e evitá-lo.

\subsection{Estabelecimento de indicadores}

Para aqueles erros com maior pontuação no GUTF, deve ser estabelecido um indicador que possa ser usado no monitoramento de sua ocorrência. Trata-se de um passo aparentemente fácil e até intuitivo, mas que, se não for bem direcionado, pode comprometer sua implantação. Assim, alguns critérios devem ser cuidadosamente observados para assegurar a pertinência dos indicadores em si bem como a adequação de sua aplicação. Neste trabalho, procurou-se orientar os gestores responsáveis que, no estabelecimento de indicadores, atentassem para que os mesmos atendessem aos seguintes requisitos fundamentais baseados nas recomendações de Neely et al. (1996): 
- Clareza: ter uma definição clara de fácil entendimento

- Utilidade: estar relacionado a um propósito bem definido

- Praticidade: deve ser de simples implantação e aplicação

- Controlabilidade: deve ser utilizável num loop de controle (feedback)

- Confiabilidade: ser à prova de falhas de medição, possibilitar verificação na fonte

- Eficiência: seu sistema de medição deve ter custo de implantação/operação viável

Tabela 2 - Critérios para a priorização das etapas (elaborada pelos autores)

\begin{tabular}{|c|c|c|c|c|}
\hline & $\mathbf{G}$ & $\mathbf{U}$ & $T$ & $F$ \\
\hline Valor & Gravidade & Urgência & Tendência & Frequência \\
\hline 5 & $\begin{array}{c}\text { Os prejuizos e as } \\
\text { dificuldades são } \\
\text { extremamente } \\
\text { graves } \\
\end{array}$ & $\begin{array}{l}\text { É necessária uma } \\
\text { ação imediata }\end{array}$ & $\begin{array}{l}\text { Se nada for feito a } \\
\text { situação irá piorar } \\
\quad \text { rapidamente }\end{array}$ & $\begin{array}{c}\text { Muito Alta } \\
\text { (1 ocorrência por } \\
\text { semana) }\end{array}$ \\
\hline 4 & Muito Graves & $\begin{array}{l}\text { Com alguma } \\
\text { urgência }\end{array}$ & $\begin{array}{c}\text { Vai piorar em pouco } \\
\text { tempo }\end{array}$ & $\begin{array}{c}\text { Alta } \\
\begin{array}{c}(1 \text { ocorrência por } \\
\text { mês) }\end{array} \\
\end{array}$ \\
\hline 3 & Grave & $\begin{array}{l}\text { O mais cedo } \\
\text { possível }\end{array}$ & $\begin{array}{c}\text { Vai piorar a médio } \\
\text { prazo }\end{array}$ & $\begin{array}{c}\text { Média } \\
\text { (1 ocorrência por } \\
\text { semestre) }\end{array}$ \\
\hline 2 & Pouco Grave & $\begin{array}{l}\text { Pode esperar um } \\
\text { pouco }\end{array}$ & $\begin{array}{c}\text { Vai piorar a longo } \\
\text { prazo }\end{array}$ & $\begin{array}{c}\text { Baixa } \\
\text { (1 ocorrência por } \\
\text { ano) }\end{array}$ \\
\hline 1 & Sem Gravidade & Não tem pressa & $\begin{array}{l}\text { Não vai piorar e } \\
\text { pode até melhorar }\end{array}$ & $\begin{array}{c}\text { Muito Baixa } \\
\text { ( menos de uma } \\
\text { ocorrência por ano) }\end{array}$ \\
\hline
\end{tabular}

Uma vez estabelecidos os indicadores a serem medidos e avaliados, a sua utilização, na gestão dos processos relacionados, requer a implantação de um sistema de informação com procedimentos especificados para a coleta dos dados necessários e geração de relatórios. Isso implica em determinar e detalhar os seguintes itens (Kaydos, 1998): a forma de cálculo (fórmula); o responsável pelo seu cálculo; a origem dos dados para seu cálculo; a freqüência de apuração (diária, semanal, mensal, etc.); e o local de registro.

\section{UM EXEMPLO DE APLICAÇÃO}

Esta seção apresenta a Fase 3 de aplicação prática do método proposto para a construção do sistema de indicadores. No caso do hospital onde o presente estudo foi conduzido, o levantamento inicial do conjunto de processos fundamentais convergiu para a definição do total de 11 macro-processos, representados no meio da Figura 4. Destes, os processos fundamentais (core) de Diagnóstico, Tratamento e Ensino e Pesquisa são os de maior importância face à missão central do Hospital. Os demais processos servem de suporte para aqueles definidos como core, sendo que o processo chamado de Liderança e Administração envolve todos os demais para Ihes dar direção e coordenação. Os macro-processos definidos englobam todos os departamentos que compõem o hospital. Na Figura 4, são apontadas as principais relações entre os mesmos e os principais stakeholders que influenciam a organização: Governo, Sociedade, Clientes e Fornecedores. 


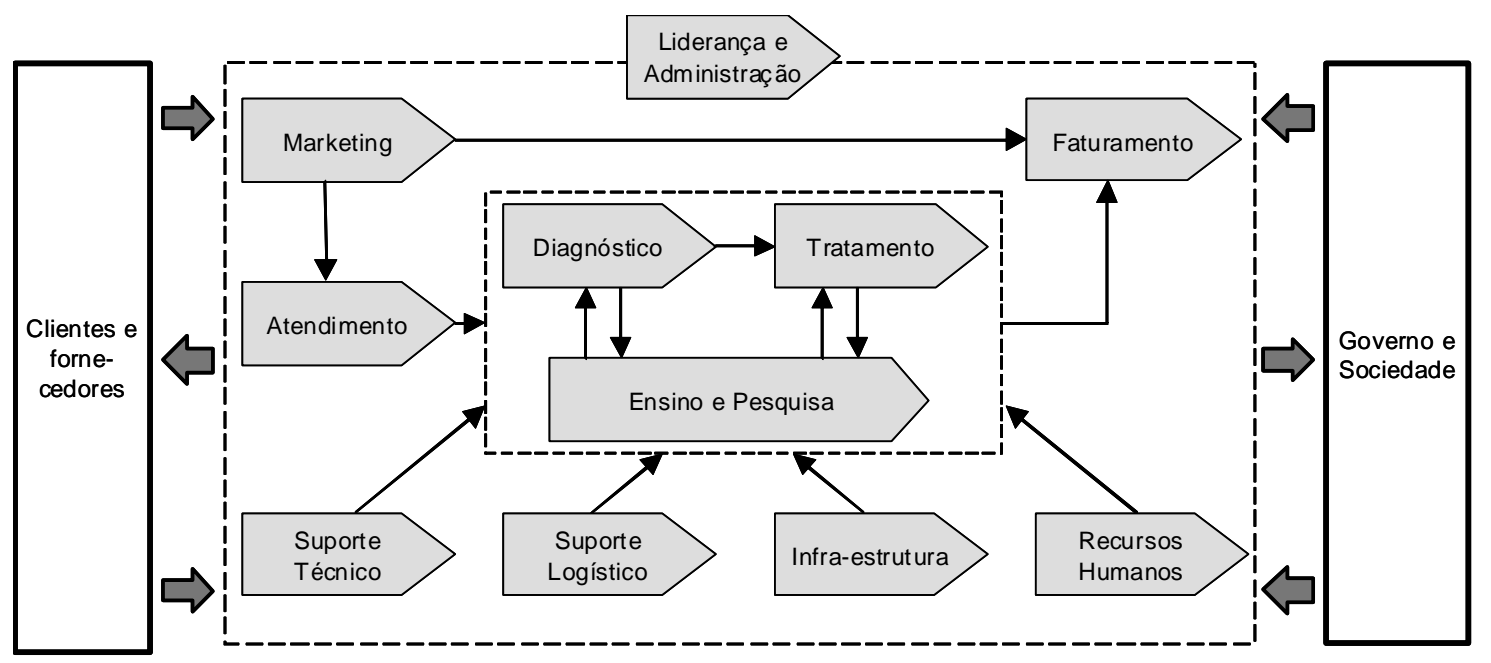

Figura 4 - Macro-processos do Hospital (elaborada pelos autores)

$\mathrm{Na}$ definição dos processos, procurou-se levar em consideração a classificação adotada no Manual de Acreditação das Organizações Prestadoras de Serviços Hospitalares (ANVISA/ONA, 2006).

Antes de prosseguir a discussão do exemplo de aplicação, é preciso esclarecer que na Fase 1 do projeto em que se insere o presente trabalho, a direção do hospital restringiu o escopo do projeto à construção de um sistema de indicadores com foco nos macro-processos considerados centrais para o hospital, deixando de fora os macro-processos de natureza administrativa ou de suporte. Assim sendo, a construção do sistema de indicadores foi fortemente orientada para o gerenciamento das atividades-fim, em que os aspectos de atenção ao principal cliente que é o paciente e, portanto, de capacitação clínica e técnica da equipe são cruciais.

Trata-se de um direcionamento que se reveste de grande significado, pois sinalizou a preocupação estratégica da direção de estabelecer um processo que possa promover a melhoria de práticas de natureza clínica. Embora aspectos como efetividade, consistência, segurança e resolutividade das práticas clínicas influam significativamente no resultado dos processos de tratamento e sejam intrinsecamente relacionados à percepção de imagem de um hospital, ainda é incomum encontrar iniciativas formais e sistemáticas de avaliação de atividades desta natureza visando a sua melhoria. Em um estudo conduzido nos Estados Unidos sobre a implantação de ações de melhoria da qualidade no setor da saúde, Shortell et al. (1998) perceberam que inicialmente tais esforços se limitaram à racionalização de atividades de natureza mais administrativa (ex. agendamento de pacientes, armazenamento de dados, cobrança) e que, somente por volta de 1994, começaram a surgir iniciativas voltadas às práticas clínicas.

$\mathrm{Na}$ condução da Fase 3, sob a supervisão dos pesquisadores, os gestores dos processos fundamentais definiram que o sistema de indicadores a ser desenvolvido deveria abranger basicamente as seguintes atividades: Atendimento ambulatorial; Diagnóstico; Exames; Tratamento por quimioterapia e/ou radioterapia; Cirurgia; Enfermagem.

O estabelecimento de indicadores para atividades de atendimento ambulatorial e diagnóstico partiu da definição de aspectos gerais que refletem a capacitação técnica com que suas atividades são desempenhadas e, em seguida, os mesmos foram traduzidos para indicadores mais específicos a cada especialidade clínica. A seguir, a aplicação do método proposto é exemplificada com 
uma descrição do desdobramento de seus seis passos até a definição final dos indicadores para uma dada atividade.

i. Identificação das principais atividades desempenhadas num dado processo

Para ilustrar sua aplicação prática, o método proposto é aqui considerado no âmbito de um processo core, o de Tratamento que envolve diversas atividades de assistência à saúde dos pacientes. A título de exemplo, é considerada mais especificamente a atividade de aplicação de uma injeção que é comum em muitos casos de tratamento que passam por este processo.

\section{ii. Definição da importância da atividade objeto de estudo}

Para os gestores responsáveis pela atividade de aplicação de injeção, sua missão pode ser entendida como "introdução de medicamentos nos pacientes, por via muscular ou intravenosa". Para saber se esta atividade foi bem executada, devese verificar o seu resultado, sendo que a ausência de complicações posteriores devidas a erros cometidos na aplicação da injeção é que indica o adequado cumprimento de sua missão.

\section{iii. Definição das etapas básicas da atividade objeto de estudo}

A atividade de aplicação de injeção é constituída das seguintes tarefas principais: preparar materiais e injeção; aspirar medicamento com a seringa; fazer assepsia no local de aplicação; aplicar a injeção propriamente dita; e fazer nova assepsia do local.

iv. Identificação dos tipos de erro que podem ocorrem em cada tarefa

Os possíveis erros ou problemas associados a cada uma das cinco principais tarefas da atividade de aplicação de injeção são enumerados na segunda coluna da Tabela 3. Neste exemplo de análise, foi levantado um total de 13 tipos de erros ou problemas. 
Tabela 3 - Possíveis falhas e sua priorização (elaborada pelos autores)

\begin{tabular}{|c|c|c|c|c|c|c|c|}
\hline Tarefa & Erro/problema & Conseqüência & $\mathbf{G}$ & $\mathbf{U}$ & $\mathbf{T}$ & $\mathbf{F}$ & Nota \\
\hline \multirow{3}{*}{$\begin{array}{l}\text { 1. Preparar } \\
\text { materiais }\end{array}$} & 1.1 Contaminação & Infecção ou reação alérgica & 5 & 5 & 5 & 1 & 125 \\
\hline & 1.2 Falta de algum item & Não aplica injeção & 2 & 3 & 2 & 2 & 24 \\
\hline & 1.3 Item avariado & Machucadura & 3 & 5 & 2 & 2 & 60 \\
\hline \multirow{3}{*}{$\begin{array}{l}\text { 2. Aspirar } \\
\text { medicamento } \\
\text { com seringa }\end{array}$} & $\begin{array}{l}\text { 2.1 Má mistura dos } \\
\text { componentes }\end{array}$ & Ineficiência do tratamento & 4 & 5 & 3 & 2 & 120 \\
\hline & 2.2 Presença de bolhas & Embolia & 5 & 5 & 5 & 2 & 250 \\
\hline & 2.3 Não limpar antes & Infecção ou reação alérgica & 3 & 3 & 3 & 2 & 54 \\
\hline \multirow{2}{*}{$\begin{array}{l}\text { 3. Fazer } \\
\text { assepsia }\end{array}$} & $\begin{array}{l}\text { 3.1 Esquecer de fazer } \\
\text { assepsia }\end{array}$ & Infecção ou reação alérgica & 3 & 3 & 3 & 2 & 54 \\
\hline & 3.2 Assepsia insuficiente & Infecção ou reação alérgica & 3 & 3 & 2 & 3 & 54 \\
\hline \multirow{3}{*}{$\begin{array}{l}\text { 4. Aplicar } \\
\text { injeção }\end{array}$} & $\begin{array}{l}\text { 4.1 Aplicar em local } \\
\text { errado }\end{array}$ & Dor ou necrose & 5 & 5 & 4 & 1 & 100 \\
\hline & 4.2 Pegar veia ou vaso & Hemorragia & 5 & 5 & 3 & 2 & 150 \\
\hline & 4.3 Injetar muito rápido & Dor & 4 & 4 & 3 & 1 & 48 \\
\hline \multirow{2}{*}{$\begin{array}{l}\text { 5. Fazer nova } \\
\text { assepsia }\end{array}$} & $\begin{array}{l}5.1 \text { Esquecer de fazer } \\
\text { assepsia }\end{array}$ & Infecção ou reação alérgica & 3 & 3 & 3 & 2 & 54 \\
\hline & 5.2 Assepsia insuficiente & Infecção ou reação alérgica & 3 & 3 & 3 & 3 & 81 \\
\hline
\end{tabular}

\section{v. Priorização das ocorrências}

Nas colunas posicionadas no lado direito da Tabela 3, são apresentadas as pontuações atribuídas pelos gestores às dimensões Gravidade, Urgência, Tendência e Freqüência para cada tipo de erro enumerado. A última coluna à direita fornece a nota que, conforme a técnica GUTF, sintetiza a avaliação geral da prioridade de se controlar cada tipo de erro.

\section{vi. Estabelecimento de indicadores}

Considerando a missão da atividade de aplicação de injeção e que seus possíveis resultados podem ser classificados como ocorrência de complicação ou ausência de complicação, foi proposto implantar indicadores voltados à medição da Incidência de Complicações (IC), com base na seguinte definição:

$$
\text { IC }=\frac{\text { Número de Complicações }}{\text { Número de Injeções Aplicadas }} \times 100 \%
$$

Para fins de priorização, a "presença de bolhas", os danos em "veia ou vaso" e a "contaminação" (itens 2.2, 4.2 e 1.1, respectivamente, na Tabela 3) foram identificados como tipos de erro ou problema com maior pontuação conforme critérios da técnica GUTF. Assim sendo, para fins de controle da atividade analisada, decidiu-se priorizar o monitoramento dos três indicadores específicos definidos na Tabela 4 visando a sua minimização. Estes indicadores complementam o sistema de medição e controle existente no processo considerado de Tratamento e possibilitam que os gestores contemplem, de uma maneira mais holística, as relações sintetizadas na Figura 3. 
Tabela 4 - Indicadores para controle da atividade de aplicação de injeção

\begin{tabular}{|c|c|c|c|}
\hline No. & Denominação & Definição \\
\hline 1 & $\begin{array}{c}\text { Presença de } \\
\text { Bolhas } \\
\text { Índice de } \\
\text { Hemorragias } \\
\text { por Dano em } \\
\text { Veia ou Vaso }\end{array}$ & $\mathrm{PB}=\frac{\text { Número de Preparações com Presença de Bolhas }}{\text { Número de Injeções Aplicadas }} \times 100 \%$ \\
\hline 3 & $\begin{array}{c}\text { Îndice de } \\
\text { Contaminação }\end{array}$ & $\mathrm{ICo}=\frac{\text { Número de Hemorragias Constatadas }}{\text { Número de lnjeções Aplicadas }} \times 100 \%$ \\
\hline
\end{tabular}

Vale lembrar que este artigo exemplifica o método de identificação e desdobramento de indicadores proposto somente para a atividade de aplicação de injeção. No desenvolvimento da Fase 3, este método foi desdobrado de forma análoga a outras atividades fundamentais dos macro-processos Diagnóstico e Tratamento, tais como realização de exames, administração de radioterapia, administração de quimioterapia, preparação de sala de cirurgia e serviço de enfermagem a paciente internado.

\section{CONCLUSÕES}

A obtenção de indicadores é um assunto relativamente recente e importante na administração de hospitais. Infelizmente, muitos ainda não têm implantado um sistema de monitoramento permanente que esteja integrado a um sistema de melhoria baseado em indicadores, ou, quando têm, limitam-se a, simplesmente, copiar indicadores que já são empregados por outros, sem a devida análise da adequação destes, nem das especificidades de seu negócio.

Como constatado no caso estudado, isso pode resultar na utilização de sistemas de avaliação e monitoramento limitados que oferecem ao gestor apenas uma visão parcial do desempenho dos processos considerados. Tal limitação pode vir a comprometer eventuais iniciativas tanto de aprimoramento de processos específicos como de desenvolvimento de novas competências organizacionais, minando tentativas de evolução rumo a patamares mais elevados de excelência na área da saúde. A promoção da visão sistêmica nas organizações hospitalares e o seu desdobramento pela via da gestão por processo jogam alguma luz sobre esta situação ao indicar um caminho que pode vir a balizar os esforços de aprimoramento da qualidade também neste importante setor.

Por outro lado, a introdução desta abordagem no ambiente real de um hospital deve ser cercada de cuidados para assegurar a sua devida compreensão e aceitação. Este artigo propôs um método de fácil assimilação e de aplicação simples para a obtenção de indicadores relevantes e alinhados aos processos fundamentais do hospital objeto de estudo. A aplicação do método proposto por meio de uma pesquisa-ação possibilitou testar a sua exequibilidade e coerência pelos profissionais do hospital que participaram do projeto-piloto para sua implantação. Longe de buscar uma abordagem mais sofisticada e inovadora, este trabalho fornece uma alternativa simples, mas eficaz por ser de fácil aceitação e por imprimir uma dinâmica bastante objetiva e assim possibilitar que seja utilizada com 
brevidade. Uma solução simples, mas implementável, é preferível a uma mais complexa e que requeira mais investimentos envolvendo assim riscos significativamente maiores.

\begin{abstract}
This paper examines the need to make the management process more integrated and effective for seeking better results in healthcare organizations in which the performance assessment practices are not yet so widely disseminated. To cope with this, a method is proposed for setting productivity and quality indicators in hospitals based on concepts of process management and systems approach. This method was applied in an action-research carried out in the context of a large hospital which has striven to become a reference center by nurturing the improvement of its service standards. The work explains how a performance indicators system should be derived starting from the identification of the organization key processes. At the end, an example is provided to illustrate how the proposed method structures the sequence of steps required for definition, selection and assessment of these indicators.
\end{abstract}

Keywords: indicators, process management, hospital.

\title{
AGRADECIMENTOS
}

Os autores agradecem a colaboração de médicos, físicos, enfermeiros e administradores do hospital em que o trabalho foi desenvolvido e o apoio do CNPq (PQ-II 307387/2007-0).

\section{REFERÊNCIAS}

Agência Nacional de Vigilância Sanitária (ANVISA), Organização Nacional de Acreditação (ONA) Manual de Acreditação das Organizações Prestadoras de Serviços Hospitalares, $4^{\mathrm{a}}$ ed., 2006.

< http://www.anvisa.gov.br/servicosaude/acreditacao/manuais/index.asp > acesso em 9/09/2008

AKAO, Y. ed. Hoshin Kanri: Policy deployment for successful TQM Cambridge: Productivity Press, 1991.

BERTO, R.M.V.S.; NAKANO, D.N. Metodologia da pesquisa e a engenharia de produção, In: ENCONTRO NACIONAL DE ENGENHARIA DE PRODUÇÃO (ENEGEP), 18. Niterói, RJ: UFF/ABEPRO, 1998.

BITTAR, O.J.N.V. Produtividade em hospitais de acordo com alguns indicadores hospitalares Revista de Saúde Pública, v.30, n.1, 1996. 
BITTAR, O.J.N.V. Gestão de processos e certificação para qualidade em saúde. Rev. Assoc. Med. Bras., v.46, n.1, Jan./Mar., 2000.

BRASIL. Ministério da Saúde. Portaria n. 1101, de 12 de junho de 2002. Estabelece parâmetros assistenciais do SUS. Diário Oficial da União, Brasília, v.139, n.112, p.36, 13 jun. 2002. Seção 1.

BRASIL. Ministério da Saúde. ANVISA. Avaliação em serviços de saúde < http://www.anvisa.gov.br/servicosaude/avalia/indicadores/index.htm\# > acesso em 9/09/2008.

BRYMAN, A. Research methods and organization studies. London: Unwin Hyman, 1989.

CECÍLIO, L.C.O. Trabalhando a missão de um hospital como facilitador da mudança organizacional: limites e possibilidades. Caderno de Saúde Pública, v.16, n.4, pp. 973-983, 2000.

CHURCHMAN, C.W. Introdução à teoria dos sistemas. Petropólis: Vozes, 1972.

COUGHLAN, P.; COGHLAN, D. Action research for operations management. International Journal of Operations \& Production Management, v.22, n.2, pp. 220240, 2002.

CROSBY, P.B. Running things - the art of making things happen. New York: New American Library, 1986.

DEMING, W.E. Out of the crisis. Cambridge, MA: MIT Press,1982.

DRUCKER, P.F. The practice of management. New York: Harper and Brothers, 1987.

EPSTEIN, M.; MANZONI, J.F. Implementing corporate strategy: from tableaux de bord to balanced scorecards, European Management Journal, v.16, n.2, pp. 190-203, 1998.

FELDMAN, L.B.; GATTO, M.A.F.; CUNHA, I.C.K.O. História da evolução da qualidade hospitalar: dos padrões a acreditação. Acta Paulista de Enfermagem, v.18, n.2, pp.213-219, 2005.

GADD, K.W.; OAKLAND, J.S. Chimera or culture? Business process re-engineering for total quality management. Quality Management Journal, v.3, n.3, pp.20-38, 1996.

HARRINGTON, H.J. et al. Business process improvement workbook. New York: McGraw-Hill, 1997.

HELLSTEN, U.; KLEFSJÖ, B. TQM as a management system consisting of values, techniques and tools. The TQM Magazine, v.12, n.4, pp.238-244, 2000. 
IBRAHIM, J.E. Quality in health care: outcomes. Best practice \& Research Clinical Anaesthesiology, v.15, n.4, pp.589-605, 2001.

JURAN, J.M. Juran on quality planning. New York: Free Press, 1988.

JURAN, J.M. A qualidade desde o projeto: novos passos para o planejamento da qualidade em produtos e serviços. São Paulo: Pioneira, 1997.

KAYDOS, W. Operational performance measurement: increasing total productivity. Florida: St. Lucie Press, 1998.

KOTAKA, F.; PACHECO, M.L.; HIGAKI, Y. Avaliação pelos usuários dos hospitais participantes do programa de qualidade hospitalar do Estado de São Paulo. Revista de Saúde Pública, v.31, n.2, pp.171-177, 1997.

LOEB, J.M. The current state of performance measurement in health care International Journal for Quality in Health Care, v.16, Supplement 1: pp. i5-i9, 2004.

MALIK, A.M.; TELES, J.P. Hospitais e programas de qualidade no estado de São Paulo, Revista de Administração de Empresas - RAE, v.41, n.3, pp. 51-59, Jul./Set., 2001.

NEELY, A.; MILLS, J.; PLATTS, K.; GREGORY, M.; RICHARDS, H. Performance measurement system design: Should process based approaches be adopted? International Journal of Production Economics, 46 47, pp. 423 431, 1996.

NEPOTE, M.H.A. Análise do desempenho das atividades no centro cirúrgico através de indicadores quantitativos e qualitativos, Revista de Administração em Saúde, v.5, n.21, pp. 21-30; Out-Dez, 2003.

ROCKART, J.F. Chief executives define their own data needs, Harvard Business Review, March-April, pp. 81-92, 1979.

SCHIESARI, L.M.C.; KISIL, M. A avaliação da qualidade nos hospitais brasileiros. Revista de Administração em Saúde, v.5, n.18, 2003.

SENGE, P. M. The fifth discipline. London: Century Business, 1994.

SHORTELL, S.M.; BENNETT, C.L.; BYCK, G.R. Assessing the impact of continuous quality improvement on clinical practice: what it will take to accelerate progress; The Milbank Quarterly, v.76, n.4, pp. 593-624, 1998.

SUMANTH, D.J. Productivity engineering and management. New York: McGraw-Hill, 1984.

TENNER, A.R.; DETORO, I.J. Process redesign. Reading: Addison-Wesley, 1997.

WILLIAMS, S.C.; SCHMALTZ, S.P.; MORTON, D.J.; KOSSS, R.G.; LOEB., J.M. Quality of Care in U.S. Hospitals as Reflected by Standardized Measures, 20022004 The New England Journal of Medicine, n.353, pp.255-264, 2005. 